\title{
Long-term follow-up of coronary MR and CT angiography for prediction of cardiac events
}

\author{
Adelina Doltra ${ }^{1}$, Ashraf Hamdan$^{2}$, Alexander Huppertz $^{3}$, Eckart Fleck' ${ }^{1}$ Sebastian Kelle ${ }^{1 *}$ \\ From 17th Annual SCMR Scientific Sessions \\ New Orleans, LA, USA. 16-19 January 2014
}

\section{Background}

Although some studies have shown the potential utility of coronary magnetic resonance angiography (CMRA) for the detection of coronary artery disease (CAD), almost no information exists regarding its prognostic value. Aim of our study was to compare the predictive value of CMRA and coronary multislice computed tomography angiography (CCTA) for prediction of cardiovascular events at long-term follow-up, with invasive coronary angiography (ICA) as the reference method.

\section{Methods}

110 patients referred to MR for suspected or known CAD were prospectively included. A CMRA at 3T, a 64slice CCTA and an ICA were performed in all patients. A luminal reduction $\geq 50 \%$ was defined as a significant stenosis. All patients were followed up for the occurrence of cardiovascular events, which comprised cardiovascular death, non-fatal myocardial infarction, necessity of revascularization and hospitalization due to cardiac events.

\section{Results}

38 events were recorded during a mean follow-up of $40 \pm$ 16 months. No significant differences in event-free survival were observed between the three techniques in the survival analysis (log-rank test $\mathrm{p}=0.989$ ) (Figure 1$)$. The area under the receiver-operator curve was $65 \%$ (95\% CI 55-76, $\mathrm{p}=0.009)$ for CMRA, 70\% (95\% CI 60-80, $\mathrm{p}=0.001)$ for CCTA and 75\% (95\% CI 66-85, $\mathrm{p}<0.0001)$ for ICA.

\section{Conclusions}

When assessing long-term prognosis, CMRA and CCTA demonstrated a similar prognostic value compared to invasive coronary angiography for prediction of cardiac

${ }^{1}$ Internal Medicine/Cardiology, German Heart Institute Berlin, Berlin, Germany Full list of author information is available at the end of the article

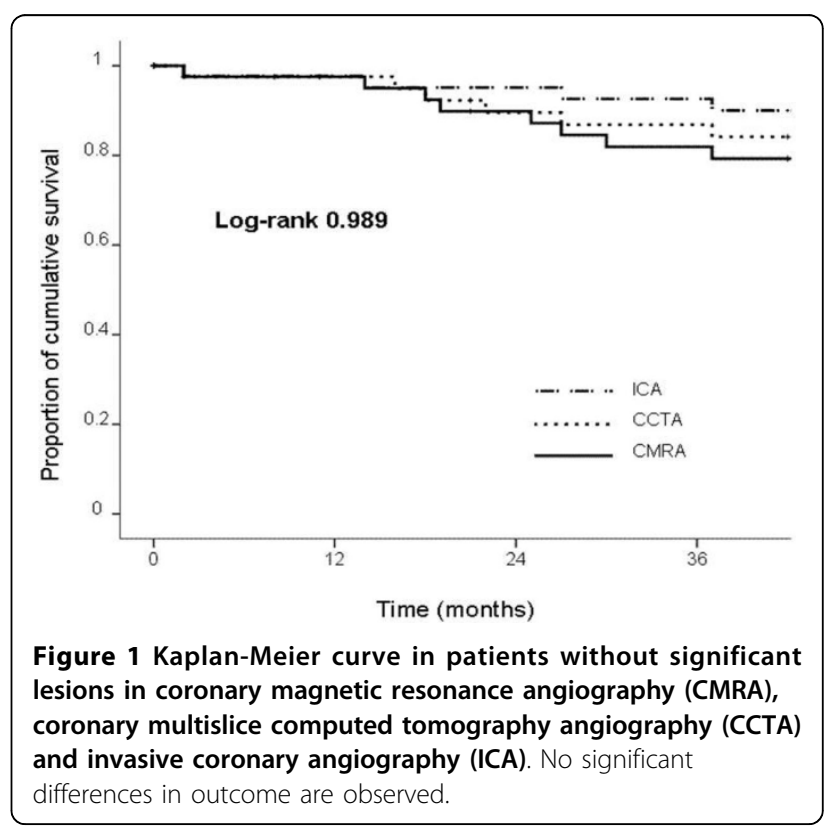

events. CMRA has a non-inferior prognostic value compared to CCTA.

\section{Funding}

None.

\section{Authors' details}

'Internal Medicine/Cardiology, German Heart Institute Berlin, Berlin, Germany. ${ }^{2}$ Cardiology, Heart Center, Chaim Sheba Medical Center, Tel Hashomer, Sackler Faculty of Medicine, Tel Aviv University, Tel Aviv, Israel. ${ }^{3}$ Radiology, Imaging Science Institute Berlin, Charité Berlin, Berlin, Germany.

Published: 16 January 2014

doi:10.1186/1532-429X-16-S1-M2

Cite this article as: Doltra et al.: Long-term follow-up of coronary MR and $C T$ angiography for prediction of cardiac events. Journal of Cardiovascular Magnetic Resonance 2014 16(Suppl 1):M2.

\section{Biomed Central}

